\title{
Quality of Learning in Ubiquitous Interaction
}

\author{
Christina Brodersen \\ Department of Computer Science \\ University of Aarhus \\ Åbogade 34 \\ 8200 Århus N, Denmark \\ sorsha@daimi.au.dk
}

\author{
Susanne Bødker \\ Department of Computer Science \\ University of Aarhus \\ Åbogade 34 \\ 8200 Århus N, Denmark \\ bodker@daimi.au.dk
}

\author{
Clemens Nylandsted Klokmose \\ Department of Computer Science \\ University of Aarhus \\ Åbogade 34 \\ 8200 Århus N, Denmark \\ clemens@daimi.au.dk
}

\begin{abstract}
Ubiquitous computing places the user in dynamic configurations of technology. As a result, learning in use has new complexities. With this paper, we aim to move beyond understanding and designing one-off interfaces by developing concepts to understand and design for learning in ubiquitous settings based on empirical examples and a foundation in activity theory. Specifically, we point to core concepts: Quality of an action; functional organs; and routines and strategies as being pivotal in analysing and designing for learning in ubiquitous settings. We argue that by focusing on general, high-quality routines we can better support learning in dynamically changing webs of technology.
\end{abstract}

\section{Keywords}

Ubiquitous interaction, learning, activity theory

\section{INTRODUCTION}

Previously, the focus of HCI has been on 'one technology-one application-one user', all packaged into a monolithic unit with a fixed set of input and output devices, and a standardized software platform, from which any task that the user would want to carry out, can be done so. Standardization, integration, and consistency framed design of such user interfaces (see e.g. Shneiderman \& Plaisant, 2005).

With the recent trends of ubiquitous interaction the user is placed in dynamic configurations of technology, where human activity is not necessarily performed through a single personal computer application, but supported by a range of technologies that supplement each other and are dynamically changing over time or through mobility. In ubiquitous interaction, the user may conduct a task using a sequence of devices, e.g. starting to work on a palmtop while being on the move; moving the task to a large screen in a meeting room, and later continuing work on a laptop. This kind of multiplicity is well-known from everyday artefacts in general: When a pilot is manoeuvring an aircraft, or the captain a cargo ship, there is not necessarily one single unified interface between the user and whatever is the object of the activity. Instead, one will see one-to-many or many-to-many relationships between the (collaborating) users, the instruments they are applying, and the objects of their activity at various levels. When looking towards learning in ubiquitous interaction, the multiplicity and diversity entail a different approach. When devices and tools are continuously and repeatedly substituted (Brodersen et al., 2007), the user should not start the learning process from scratch with each new device. At the same time, the old mantra of "keep it simple, stupid" is neither the goal. Users should be able to apply their domain knowledge, their knowledge of use with similar devices, and their understanding of humancomputer interaction in general to the device.

When designing ubiquitous interaction it is essential to understand how the multiple devices supplement each other, while making the best possible use of the capacities of each and every device. Accordingly, it is no longer evident that standardisation, integration and consistency are the main design criteria. We urge designers to assume and utilize the competences of the users, and not think their designs in isolation. In this paper, we suggest an understanding of learning in ubiquitous interaction focussing on general high quality routines to support interaction in dynamically changing webs of technology better.

\section{THEORETICAL BACKGROUND}

Learning, in general, is the development of operations, actions and activities (and transitions between them) as they take place in use, or in dedicated learning activities. Along with Bødker \& Petersen (2000), we focus on the learning that takes place in use of instruments, between users of instruments and in the transfer of experiences that users make between artefacts. In more classical human-computer interaction settings, we would look at the learning that takes place in a well-established community of users, surrounded by manuals, training courses etc. Hence, the learnable instrument may encompass the models, references to other instruments, etc. that are part of the web of activities of the users. This is less so in design for ubiquitous interaction qua its changeability. In Brodersen et al. (2007) we combine the analysis of computer-mediated human activity (Bødker 1991, and Bertelsen \& Bødker, 2003), with an extended notion of affordance (Bærentsen \& Trettvik, 2002); Kaptelinin's development of the notion of functional organ (Kaptelinin, 1995), and Bedny et al.'s (2000) concept of strategies as plans for goal achieve- 
ment. Figure 1 shows the relation between the three levels of activity and the descriptional focus on strategies and routines (see further in Brodersen et al, 2007).

\begin{tabular}{|c|c|c|c|c|}
\hline $\begin{array}{l}\text { Levels of } \\
\text { activity }\end{array}$ & Mental representation & Realizes & Level of description & $\begin{array}{l}\text { Analyti- } \\
\text { cal } \\
\text { question }\end{array}$ \\
\hline Activity & 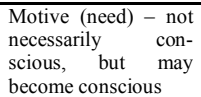 & Personality & $\begin{array}{l}\text { The social and } \\
\text { personal meaning of } \\
\text { activity, its relation to } \\
\text { motives and needs }\end{array}$ & Why? \\
\hline Action & Goal - conscious & $\begin{array}{l}\text { Activities } \\
\text { (systems of } \\
\text { actions } \\
\text { organized to } \\
\text { achieve goals) }\end{array}$ & $\begin{array}{l}\text { Possible goals, critical } \\
\text { goals, particularly } \\
\text { relevant sub-goals } \\
\text { Strategies }\end{array}$ & What? \\
\hline Operation & $\begin{array}{l}\text { Condition of actions } \\
\text { (structure of activity) } \\
- \text { normally not } \\
\text { conscious, only } \\
\text { limited possibilities } \\
\text { of consciousness }\end{array}$ & $\begin{array}{l}\text { Actions (chains } \\
\text { of operations } \\
\text { organized by } \\
\text { goals and } \\
\text { concrete } \\
\text { conditions) }\end{array}$ & $\begin{array}{l}\text { The concrete way of } \\
\text { executing an action in } \\
\text { accordance with the } \\
\text { specific conditions } \\
\text { surrounding the goal. } \\
\text { Routines }\end{array}$ & How? \\
\hline
\end{tabular}

Figure 1 The hierarchy of human activity

Kaptelinin (1995) captures the functionally integrated, goal-oriented configurations of internalized operations and procedures (routines and strategies), and external mediation in the term functional organ. When a mediator is well integrated into the functional organ it augments the human capacity, whereas if not, the mediator is outside the human user. In Brodersen et al. (2007) we conclude that a well-integrated functional organ fits on four analytical layers (see Figure 2), whereas understanding problems of the integration sheds focus on discrepancies at either layer, or across layers.

\begin{tabular}{|l|l|l|}
\hline Analytic layer & \multicolumn{2}{|c|}{ Functional organ } \\
& Mediator/external & Routines/internal \\
\hline Need-related-why? & Need-related aspects & Motivational routines \\
\hline Instrumental-what? & Instrumental aspects & Actions and strategies \\
\hline Operational-how? & Operational aspects & Operation and routines \\
Handling & - Handling aspects & - Learned handling \\
Adaptive operational & - Adaptive aspects & - Adaptation \\
\hline
\end{tabular}

Figure 2. Functional organ

We distinguish between strategies that are consciously developed to perform an action, and routines where the predisposition to perform a certain action is partly automated or non-conscious. Human beings initially deploy strategies. Through execution and repetition of these actions under various material conditions, routines get developed, and the repertoire of action possibilities becomes richer (Bedny 2000, Bødker 1991). In ubiquitous interaction, the user cannot rely completely on the specific development of routines for each artefact, as the different configurations of artefacts are virtually endless. Accordingly, we need to focus on the quality of the actions and their connected routines. In addition, the adaptation of routines to sound strategies for interaction across artefacts need to be explored further.

The Quality of Ubiquitous Interaction

Gal'perin (1969) judges the quality of an action through the levels of

- generality (i.e. to recognize properties of an object relevant to complete an act),
- abbreviation (i.e. to consciously skip operations in the performance of an act) and

- mastering (i.e. to independently repeat an act with new material) achieved within the action.

Central to supporting transfer of actions and routines between material conditions is the type of plan or orienting basis. Gal'perin (ibid.) distinguishes between three types of orientation:

\section{- Trial and error}

- Reusable knowledge within a specific domain

- Domain independent general methods

In the trial-and-error type, the user searches, without help from established routines, between the possible conditions, and learns to distinguish between the correct operations as the different possibilities are tested. Through trial and error, the user learns to perform one specific task (where the setting is stable), but transfer to other tasks in other settings is highly limited because the routine lacks the necessary generality.

Re-usable knowledge within a specific domain is characterized by a much higher degree of guidance of the learning process, and a deconstruction of the problem into separate operations that support the users' ability to analyze and handle the situation. Transfer between tasks within the domain is significant, and relies on the similarity of material conditions, purposes and artefacts. However, routines do not necessarily transfer to domains, and the performance is therefore restricted to the specific domain. Thus, each new domain requires the development of a new orienting basis.

General methods allow the user/learner to develop an orienting basis independently of any specific act or assignment. General methods may be learned through a more complex type of instruction that go beyond the concrete phenomena, at the same time as they allow experimentation with this. Such learning allows the user to gain an understanding of the underlying structure of the problem (a theoretical generalization), in addition to working with concrete instances of it. This type of orienting basis is very stable under changed conditions, meaning that transfer is virtually unlimited within the given field. Furthermore, the user can direct his attention towards the model for keeping track of the progress of the concrete task, and this supports abbreviation, i.e. consciously condensing the task by "skipping" ahead in the line of operations necessary for achieving the goal.

The ability to conceptualize a routine for performing a task into a strategy for performing another relies heavily on the orienting basis, and how the routine has been developed in the first place. If the routine has been developed through trial-and-error, there is less chance that it can be reused to develop sound strategies for other tasks, since fundamental understanding might be missing. In Gal'perin's terminology, routines can be abbreviated either consciously or unconsciously. Conscious abbreviation is natural and non-harmful whereas uncon- 
scious skipping of intermediate results or actions because of lacking knowledge can be extremely harmful.

Bardram \& Bertelsen (1995) use Gal'perin's work to focus on learning in use, and Bødker \& Petersen (2000) extend this focus on the learnable artefact. None of these papers deal specifically with the challenges of ubiquitous interaction. Bardram \& Bertelsen (1995) introduce three dimensions of the learnable/transparent interface that are developed further in (Bødker \& Petersen, 2000):

- initial familiarity;

- formation of new operations; and

- development in use.

When we enter the area of ubiquitous interaction that is characterized by dynamically changing conditions, it becomes necessary to move beyond trial-and-error. We need to concern ourselves with how we may heighten the quality of the interaction through the support of mastery, abbreviation and generalization in the design of the devices and interfaces used in the materialized stage. Using initial familiarity, formation of new operations, and development in use as key concepts for analysis and design presents us with strong tools for understanding the learnable, ubiquitous interface.

\section{UNFOLDING THE LEARNABLE INTERFACE}

We move on to unfold the three dimensions of the learnable interface. In doing so, we use empirical examples to illustrate and discuss our concerns for quality of learning. Hence, they shape the theoretical concepts and discussions, not vice versa. The empirical material precedes these theoretical discussions.

We use a study of interaction with three map-devises to explore the dimensions of learning in a ubiquitous setting. The map-study (Textbox 1) is particularly useful for this purpose, because it focuses on what happens when people are asked to use several map-devices after each other. Furthermore, the map study helps us address differences and similarities resulting from expertise in map reading and knowledge of the local area.

The wastewater example (Textbox 2) supplements this insight with a focus on design of multiple, ubiquitous interfaces. Wastewater treatment is a highly explorative process, and the section discusses how ubiquitous interaction may be designed to reflect this.

\section{Textbox 1. The Map Study}

In Bouvin et al. (2006), we presented a study of the use of maps on three different technologies: A paper map in a book, a digital map on a tablet-PC, and a digital map on a Nokia smart phone. The study was performed with six groups with 2-3 members, all adults. Each group was given eight assignments-e.g., locating north, or identifying a local school and the distance and direction to it-half to be done with one technology, half with another. All assignments relate to objects that are (more or less) visible through a large window in our building. The focus for the study was to analyse and understand how users solve similar tasks across heterogeneous devices.

\section{Initial Familiarity}

With the perspective presented above, initial familiarity is obviously very important. Initial familiarity of an artefact sets the stage for the users' ability to recognize the potential of integrating it as a functional organ, and curtail the level of trial-and-error the users have to resort to in order to appropriate the artefact. Our hypothesis is that this is done by recognition on one or more of the analytic layers of the new artefact, and triggering in the user a set of routines and procedures to match these. Bearing in mind the four aspects of functional organs, initial familiarity includes recognisability of all four analytical layers of activity: Motive and purpose (why and what) as well as handling and adaptation possibilities. The initial familiarity can trigger strategies bound to the specific domain or from more general knowledge, and formation of new operations can be based upon these. Accordingly, the initial familiarity is both dependent on immediate similarity with other artefacts, and on the quality of the actions of the users before they enter the situation.

The map study sets a scene where participants knew that they were handed a map-device, and hence, the motive and purpose were largely given by the assignment. As pointed out in Brodersen et al. (2007), the users drew upon routines and knowledge stemming from three sources or domains: The local neighbourhood that they looked at, where some group members walked or biked daily and made reference to; maps, e.g. dealing with scale, road names, direction, etc.; and the device, e.g. leafing pages or using the pen on the tablet.

\begin{tabular}{l|l|}
$\begin{array}{l}\text { B: the big red thing } \\
\text { that's a church }\end{array}$ & $\begin{array}{l}\text { B looks out } \\
\text { A looks at the map } \\
\text { A: ... it is a school } \\
\text { B: aaah ... there must } \\
\text { be a church there }\end{array}$ \\
$\begin{array}{l}\text { A: Let's see. Christian- } \\
\text { skirken }\end{array}$ & map search the
\end{tabular}

Figure 3. Using a paper-map together

All groups recognized the paper-map immediately. None of the groups showed any hesitation in recognizing they were given a map of the neighbourhood that they were located in, and all groups proceeded to identify their own location (Figure 3). At this point, however, there were clear differences between the groups. Most of them understood the grid ${ }^{1}$ structure common to paper maps, and used it to identify which page on the map to leaf to. At least one group, however, had no understanding of the grid system, and resorted to a trial and error-exploration of the map pages.

In Brodersen et al. (2007) we point out how several groups use general strategies for finding a location, independently of grid or zoom. One group was particularly artistic, however, applying a routine of measuring across two devices: They measured a known distance using a piece of paper and two fingers as yardstick.

\footnotetext{
${ }^{1}$ The division of a map into numbered zones using a grid.
} 
When handed the tablet-map, all groups were told that the pen used for interaction functioned like a mouse. This was enough for most of the groups to start using the map, and to pan and zoom to find their own location. However, not all groups found it immediately easy to measure distance on the tablet; when these groups were asked to measure distance, the 'pen-as-mouse'interaction caused problems: The pen was seen as a pen (Brodersen et al., 2007), and some groups tried to measure distance by drawing, rather than clicking at end-points. At least one group did not manage to use the tablet for measuring distance. This 'false' initial familiarity triggered routines not usable on the tablet.

Of the three devices, the cell-phone map is the most challenging in terms of understanding how it supports initial familiarity. The map itself is the least useful of the three maps, because it is very limited in size, and is slow in panning/loading new map fragments. For almost all groups this meant that they gave up looking at the map, and relied entirely of their area knowledge and view out the window in answering questions. In one example, the group had been quite good at establishing a mapping between the paper map and the view through the window. However, after switching to the phonebased map, they immediately stopped any attempt to use the map as an aid in finding the direction.

The cell-phone map is interesting because our user groups seem to range from users who already have wellestablished general procedures as regards cell-phones, to users who have barely held a cell-phone before. The initial use of the phone was therefore based on either an understanding of map-use and only weak understanding of a cell-phone, or understanding of both. During one task, the group members used routines for interaction with the phone developed in everyday use of cellphones. They focused on the task and the feedback. The interaction with the cell-phone was fluid and unencumbered and the understanding of the menu structures and general information hierarchies associated with cellphones was clear. The group members expected to find a distance-measuring tool on the phone and started looking for it by browsing through the menus. However, this feature did not exist and, even though both the cellphone and the map had become functional organs for the group members, the combination of the two caused problems and what seemed to be initial familiarity with the phone-map turned out to be a dead end.

Initial familiarity can derive from multiple aspects of a mediator. In the map study, we saw how the familiarity can be constructive, e.g. most of the groups' unhindered use of the paper map, and most groups' ability to easily pan and zoom on the tablet-PC, however counterconstructive when false. Designing for initial familiarity in the case of the kind of map use addressed here, would require providing a decent overview with the ability for zooming and panning, interaction true to the provided input device, and considerations in the design of what kind of functionality will be expected given the users familiarity with the device.

\section{Formation of Operations}

Understanding and designing for formation of new operations addresses two further elements of use: To support the development of more elaborate routines/operations, and the development of a richer set of alternative routines. Formation of operations also means to form quality actions at more elaborate levels. Hence, generality includes a concern for how the appropriate operations among alternatives get triggered. Abbreviation must be allowed for, or facilitated, in terms of users understanding redundant steps to be skipped, while not being led into skipping significant ones. Mastering independently with new material relates to the generality of the operations being formed, and to the ways in which experimentation and trial-and-error finds its place in the repertoire of operations-as controlled ways of taking in e.g. new materials, rather than as total breakdown based on inappropriate routines.

The 'false' pen caused extensive trial and error interaction by one group, specifically resulting in drawing numerous lines on the map when attempting to measure distance. The group also panned in rather uncontrolled ways while still in the process of measuring the distance. However, at some point, and seemingly at random, they clicked with the pen and got feedback to indicate that they were measuring, and from then on measuring stopped being a problem.

Furthermore, the same group made an early wrong identification of the orientation of the map; north was off by approximately 45 degrees. For a number of assignments, group members used this flawed identification because they trusted their initial determined direction, and quickly abbreviated without checking it again. It was not until their very last assignment, and after having changed map device, that they realized they had made a visible identification that would have to be located behind them, according to their own previous identification of north. While this abbreviation was not directly related to the map, it seemed to be connected to how they used, or rather gave up using, the cell-phone map. In general, all groups 'using' the cell-phone map did not use it unless they absolutely had to: They guessed, tried to remember, looked out the window, and used experiences from previous exercises as much as possible. We see this as a result of the very limited size of the cell-phone map view (Brodersen et al. 2007).

In this setting, almost all of the users had never used maps on tablet-PC and cell-phone before. Their formations of new operations were therefore heavily based upon their degree of initial familiarity. Hence, their problems in forming new operations were mainly connected to problems with initial familiarity. Abbreviation of operations where users used fingers as bookmarks was extensively used on the paper map, and to some degree on the tablet-PC. On the paper map, previous distance measurements were used as the basis for performing new ones. If we were to redesign the map on e.g. the tablet-PC based on the experience from the map study, some implementation of a book-marking tech- 
nique would be appropriate, hereby making the users capable of abbreviating repetitive task, such as refinding home or target.

\section{Development in Use}

Where formation of operations is about the development at the operational layer, development of use is a matter of setting up conditions in the artefact that make users apply them beyond their current (conscious) needs and motivation, i.e. the instrumental and need-related layers. Hence, artefacts that aim to support development in use must be challenging the taken-for-granted motivational routines of the users (while, obviously, still supporting initial familiarity).

Evidently, the map study contains limited examples of development in use, since the groups use the mapdevices only briefly. Nonetheless, we do see what we could call seeds (Engeström, 1987) of such development in some of the situations. One group recognized the tablet-PC as a PC as well as a map. When they realized that they could not identify buildings on the tabletmap, they started to look for solutions outside the map. They realized that they could get access to a search engine in a web-browser. However, they lacked inputpossibilities, and proceeded to look for a soft keyboard. Once that was found, they looked up the address of the school they were looking for, and as they had 'unlocked' new action possibilities, they proceeded to look up more precise answers to several of the assignments they had already completed. Another group, as mentioned earlier, failed to identify a way of measuring distance on the cell-phone. At one point, they decided to investigate if the menu system of the cell-phone would help them, and they explored the phone, rather than the map. The group experienced a breakdown despite their high degree of familiarity with the technical device, and knowledge of maps in general. Their expectations of the functionality simply exceeded what was actually offered by the cell-phone map. Accordingly, a further development of this particular kind of use was prevented due to a clash between functional organs: On one hand, being used to measure distance on the map, and on the other using the computational power of the cell-phone.

Whether successful or not, the kind of development in use found in the map study stemmed from the users combining their knowledge of the map, the assignment and the capabilities of the device to realize new possibilities. And, though the study was limited, it seems clear that a redesign should allow users to move from

\section{Textbox 2. Wastewater Plant Study}

In this study (presented in Bertelsen \& Bødker, 2001, Bertelsen \& Bødker, 2002, Nielsen \& Søndergaard, 2000, Bertelsen \& Nielsen 1999), field studies and interventionist approaches were carried out to develop new computer support for the running and optimisation of a wastewater plant. The study was part of a long-term research co-operation in the areas of $\mathrm{HCI}$ and CSCW involving Danfoss and the Computer Science Department at Aarhus University, as well as several other partners. The purpose is to explore the theoretical notion and practical design of common information spaces. A prototyping experiment was conducted, focusing on how to get and maintain a local overview of parts of the plant while moving about, and on compiling and interpreting information that is massively distributed on meters and dials (Bertelsen \& Bødker, 2001). the map to other functionalities available on the particular device (and as such, these other functionalities should be visible to the users). At the same time, however, it should be possible for users to 'return' to the map, and bridge between the uses and experiences. To avoid the clash of models we saw with the cell-phone, it is an important part of a redesign to aim for a wider, expandable model of the new map-device.

\section{DESIGNING THE LEARNABLE ARTEFACT}

We will now move on to the wastewater case, to show how concerns for learning across interfaces can shape the design of prototypes. Oversimplified, the process of wastewater treatment deals with the extraction of unwanted elements from sewer water, in order to produce water clean enough for human consumption. Work at a wastewater treatment plant includes an act of balancing elements of mechanical, chemical and biological purification, while keeping down the cost of the purification process. The wastewater operators were constantly optimizing the process to produce cleaner water, increase the throughput of the plant, etc. Furthermore, they were constantly engaged in tasks to maintain the different components in the plant. In the following, we will identify key elements and routines of the wastewater operators' work, and give examples of how the highlevel goals (optimization and trouble-shooting) were translated into the design.

A typical use scenario looks as follows: The wastewater treatment process is managed by the central control and regulation system, located in the control room. However, none of the workers spend more than five or ten consecutive minutes at a time in that control room. They work outside the room, outdoors, or in other buildings of the plant where their daily routines depend on having direct access to the local environment and the components in it. The specific locations at the plant, like the primary clarifier, provide the workers with sensory information not measurable by the automatic sensors, e.g. the way the water look and smell. As part of his daily round, Bob is checking the area around the sludge tanks, and notices that the surface water in the tanks is brownish. He immediately proceeds to check a pump, which he, rightfully, suspects to be malfunctioning. The sensory clues prompt Bob to alter his routine.

\begin{tabular}{|l|l|} 
Analytic layer & Routines/internal \\
\hline Need-related-why? & Optimizing, trouble shooting \\
\hline Instrumental-what? & $\begin{array}{l}\text { Sampling automated sensor readings, manual readings, e.g. } \\
\text { lab results. Monitoring control system }\end{array}$ \\
\hline Operational-how? & $\begin{array}{l}\text { Operation and routines } \\
\text { - interpreting smell, looks, etc. } \\
\text { - adding sensor readings to control system, reading controls }\end{array}$ \\
- smelling, looking, moving about \\
- interacting with control system and individual components
\end{tabular}

\section{Figure 4 Existing routines to be accommodated}

This scenario gave rise to a number of design challenges: Local sensory information along with information from the control system, formed the basis for how work was performed, and in most cases, any one source was not sufficient for deciding how to progress. However, the information obtained from walking around in 
the area was completely detached from the information from e.g. the sensors, which were only available through the control system. This made the task of getting an overview of the 'state' of the process extremely complicated. Figure 4 provides an overview of the tasks and routines connected to the high-level goals of optimisation and trouble-shooting. We proposed two prototypes, on a PC and a PDA respectively, to support optimisation and trouble-shooting by providing a better overview of the purification process at different levels of detail. The intention of the two prototypes is to support users in being out in the plant, walking and using ones senses, while having some access to the control system. At the same time they are able to work in the control room with the extended functionality there.

Figure 5 shows the two topmost levels of detail. First is the plant overview, where the entire purification process and the corresponding sludge and gas processes are represented in the same abstract form in both prototypes. We chose a simple geometric shape for both platforms, without completely overloading the PDA. At the same time, we wanted to avoid designing for the lowest common denominator by extending the PCversion with a "linking space" to the left, where the user could set up relevant links to other parts of the system, in combination with the control system overview. Second is an overview of a local area, in this case, the inlet, with available sensor data. At this level, focus is on getting an overview of the different components available to the wastewater operator. The circumference of the circle represents the max limit for each sensor, which is easily interpretable, despite the fact that the units for the individual sensors differ significantly.
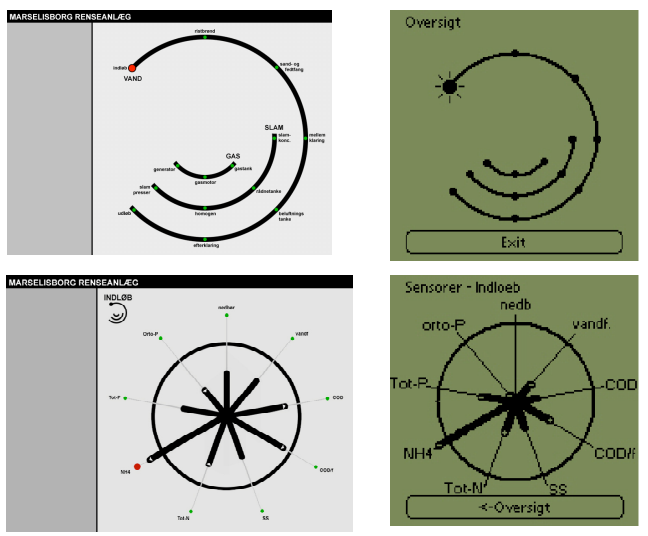

\section{Figure 5 - Plant overview. First and second level}

The design idea is that using the same graphical representation for a collection of different devices allows the wastewater operators to bring along a 'window' into the control system when they leave the control room, and combines the two hitherto independent information spaces without compromising the overall security of the control system. The strong graphical similarity across platforms aims to ensure that the wastewater treatment process is recognized as the overarching structure within which tasks are performed, and information relating to the same parts of the plant is relating to the same parts of the plant is recognizable because of their graphical similarities. These prototypes were evaluated with wastewater operators in workshops as described in Nielsen \& Søndergaard (2000). These experiences along with the design as such will form the basis for our exploration.

\section{Designing for Initial Familiarity}

Designing for initial familiarity means creating a design that supports and takes advantage of the strategies and routines possessed by the user, with respect to the tasks and domain. We drew upon the wastewater operators' vast understanding of the wastewater treatment process, in the creation of the prototypes. By focussing on overview of the plant processes, and possibilities of zooming in on information, we made use of the understanding of the wastewater workers in terms of why, what and how.

In the prototypes, we used the terminology known from the current control system to support recognition despite the radically different interface; the elements used to represent key points in the wastewater purification process were taken from their 'old' system, but restructured to represent an abstract version of the process. Furthermore, using a minimalist approach to building the interface on both devices-the simple geometric shapes, the colour and the sparse textual informationhelped the wastewater operators sort between the available properties. However, on the PDA, the lack of textual cues on the first level proved to be too minimalistic; the wastewater operators had to rely on their knowledge of the purification process, and what they remembered from the PC-version to find the right information on the PDA. Hence, they quickly reverted into a trial-and-error pattern of use. In a sense, wastewater operators were forced to guess how we had interpreted the wastewater purification process and embodied it in the design.

Designing for initial familiarity, in a case like this, means to take the existing routines seriously. Despite the fact that the graphical user interface was radically different from what the wastewater operators were used to work with in the control system, they had no problems recognising the structure and different elements of the wastewater treatment process in its abstract form. They quickly learned to navigate through the different prototype levels, and asked questions about where to find additional information about key elements in the process. Their feedback strongly indicated that they found this structure to be superior to the existing presentation of the wastewater treatment process with respect to maintaining an overview, as well as being very useful for explaining the purification process to others, for example visiting school children. This latter point is an important indication that they recognised the prototypes as something that would help them beyond their own sampling of data, towards an understanding of wastewater processes that was more general. This is well in line with how the wastewater operators see themselves as part of an ongoing experiment to optimise the running of the wastewater plant (Bertelsen \& Bødker 2001). This perspective is discussed further below. 


\section{Designing for the Formation of New Operations}

Designing for the formation of new operations means creating a design that supports the development of more elaborate routines and operations, as well as a richer set of alternative routines. To achieve this, the design must support optimisation and trouble-shooting in a way that does not prescribe a restricted set of routines bound to the different analytical layers of the functional organ. The work of wastewater operators is characterised by being highly unpredictable, because they often have to respond to unexpected events (trouble-shooting). Thus, the interaction with the control system and the individual components must be flexible enough to adapt to the changing circumstances, and their repertoire of routines and operations must be general and mastered over a wide set of conditions. However, despite our efforts to support a high degree of flexibility, we realised the structure of the prototypes was flawed; the design allowed users to move one level up or down, but it was not possible to jump across several levels in the information hierarchy. This restriction made it difficult for the wastewater operators to create navigational shortcuts, and thus to consciously skip operations.

This is an example of a lack of possibilities for abbreviation in the prototypes on the instrumental level of the prototypes, which would affect the need-related aspects of the use of the prototypes. It presented a hindrance of the formation of new operations on the two devices, and must, naturally, be supported better in both prototypes. Without it, it is doubtful that the prototypes would become functional organs to the wastewater operators. Thus, designing for the formation of new operations in this context puts focus on the importance of combining an understanding of the work practise and tasks, with an understanding of the technological possibilities because we, to some degree, design for unanticipated use. This example also illustrates how ubiquitous devices are rarely singular. Obviously, we chose to design a family of interfaces, but in doing so we also brought out a focus on the differences and boundaries between the two: The similar graphical layout appeals to the similarities of use between the two, but nonetheless, it is important that the users understand and operationalize the differences, so that they have a set of routines for both. A set of routines that gets appropriately applied when facing the "right" interface, and that includes the operationalized choice between the two. We aimed to support "telling the difference" through the different degree of sophistication, in terms of use of colour and resolution, and through the different screen use. We have no real evidence to show if this worked in the long run, but we find this an interesting design challenge when designing multiple interfaces in general.

\section{Designing for Development in Use}

We have already pointed out that wastewater treatment is by its very nature highly experimental and unpredictable (Bertelsen \& Bødker 2001). To design ubiquitous artefacts that support development in use seems well in line with this unpredictability, and focuses on ways of challenging the taken-for-granted motivational routines of the users while obviously still supporting the initially familiarity at all levels of activity.

In the PC prototype, the 'linking space' encourages users to mix information from other parts of the system as the need arises. The wastewater operators can build their own information space, in relation to the view provided of the wastewater treatment process. The PDA version does not have this linking space access, because the tasks it was to be used for are highly local, dealing primarily with maintenance and trouble-shooting at specific components. With the prototype design we tried to present an explicit model of the wastewater treatment process at the same time as we allowed the wastewater operators to work on specific components. They would maintain the overview of the process and the individual components. The similarities across the user interfaces were to support an orienting basis that was general enough to include both interfaces, and a high degree of transfer of routines between the two.

The focus on development in use and challenging of existing routines made us aware of more areas not originally thought of as relevant for the prototypes, and hence were not supported. For example, it should be possible on both devices to add and remove sensors from the different points of interest in the process. This would allow users to re-use knowledge about, and repertoires of, operations on sensors while applying this understanding to new conditions.

The example of how wastewater operators were imagining using versions of the new design with visiting school children, represents the seeds of an entirely new type of use: Visualizing the processes of wastewater in a pedagogical setting, while moving about the plant.

Since optimization and trouble-shooting are such essential parts of wastewater treatment, it is particularly challenging to design instruments that allows for experimentation, and unanticipated use as well. The overall design that runs across the two sorts of devices has all the way through been focusing on generality of actions, possibilities of formation of new operations, while emphasizing the mastering across material conditions and across the two interfaces. The simple visual design was an important part of emphasizing learning and exploration across interfaces. Unanticipated by designers, it gave rise to possibilities of adding and removing e.g. sensors in a manner that supported transfer of handling and understanding across plant components. How such adding (and removal) of sensors would become a part of the everyday handling of the two overview-devices is a question that could well be explored as an issue of tailoring or end-user programming. Addressing end-user programming from the learning perspective presented here is a further challenge for design.

\section{THE LEARNABLE ARTEFACT IN SUMMARY}

In this paper, we have addressed the quality of learning in the context of ubiquitous interaction. Ubiquitous interaction differs from classical one user-one device- 
one application interaction, as being interaction with multiple, and changing configurations of devices and settings. Hence, long-term learning processes with a single device in isolation cannot be assumed. Learning should transcend the isolated device, and rely on the use and development of high-level high-quality routines. High-level high-quality routines are routines applicable across devices, and perhaps even across domains. Though imprecise as measuring tool, the measuring of distance between two fingers, or using a sheet of paper to mark a ruler, are examples of this.

Central for designing learnable artefacts in the context of ubiquitous interaction, is supporting of transfer of use between artefacts-not designing for use of the artefact in isolation. In this paper we have argued how it is important to support reuse of high-level routines, rather than relying on trial-and-error alone.

Trial-and-error however is a natural part of any learning process. It would be naïve to assume that trial-and-error could be completely avoided. What is import though, is that either domain-specific routines or general routines frame the trial-and-error. Blind trial-and-error occurs either when the user has no clue of how to approach the interaction (they may not understand what the artefact serves for or why), or when the general routines, or the domain specific routines drawn from experience, are not applicable to the specific device. Central for the learnable artefact is that trial-and-error is limited to the lowlevel adaptation of a high-level routine to a specific artefact; e.g. being able to adapt the routines of navigating to a map, or the knowledge of the structure of a wastewater plant to a new artefact, without breaking with these routines and resorting to blind trial-and-error.

One of the challenges in designing the learnable artefact, is making the users initial familiarity trigger the appropriate high-level routines, and avoiding to trigger 'false' familiarity as e.g. in the case of expected but lacking distance measuring on the cell-phone. The learnable artefact should further exude its capabilities, so a user in a ubiquitous interaction setting will be able to chose what device to use when, and for what.

Just like trial and error is a natural part of learning, so is the focus and understanding of the domain. While we argue for simple and general artefacts, we would not get far in modern society if we only designed for totally generic artefacts. Central to the learnable artefact is that it takes its starting point in a purpose, a motivation and the reusable knowledge and routines within the domain. This sets the stage for trial-and-error exploration as well as for identifying the possible general routines to be supported in design. At the same time ubiquitous interaction takes place in dynamically changing webs of technology and activities. These webs means that domains of knowledge and understanding in the ubiquitous interface design stem from many overlapping arenas of life-from wastewater optimization, to cell-phone use-which can be 'activated' trough the appropriate design choices. Accordingly, it is essential to design for sustainability and adaptability of routines beyond the specific use situation. We propose that it is in this space that we look for quality of actions.

\section{REFERENCES}

Bardram, J. E. \& Bertelsen O. W. (1995). Supporting the Development of Transparent Interaction. In Blumenthal, B., Gornostaev, Y. \& Unger, C. (Eds.). EWHCI '95 Selected Papers. Berlin: Springer Verlag, pp. 79-90.

Bedny, G. Z., Seglin, M. H., \& Meister, D. (2000). Activity theory: history, research and application. In Theoretical Issues in Ergonomics Science, 1(2):168206.

Bertelsen, O. \& Bødker, S. (2002). Interaction through multi-artifacts. In Bagnara, S., Pozzi, S., Rizzo, A. \& Wright, P. (Eds.). ECCE 11 - Cognition, Culture and Design, pp. 103-111.

Bertelsen, O. \& Bødker, S. (2001). Cooperation in massively distributed information spaces. ECSCW 2001, Dordrecht: Kluwer Academic Publishers, pp. 1-18.

Bertelsen, O. W. \& Bødker, S. (2003). Activity Theory. In Carroll, J. (Ed.). HCI Models, Theories, and Frameworks: Toward an Interdisciplinary Science, chapter 11. San Francisco, CA: Morgan Kaufman Publishers, pp. 291-324.

Bouvin, N. O., Brodersen, C., Bødker, S., Hansen, A., \& Klokmose, C. N. (2006). A comparative study of map use. In CHI '06 extended abstracts, ACM Press, pp. 592-597.

Brodersen, C., Bødker, S., \& Klokmose, C.N. (in press 2007). Ubiquitous Substitution. In press for Interact' 07.

Bærentsen, K. B. \& Trettvik, J. (2002). An activity theory approach to affordance. In Proceedings of NordiCHI 2002, ACM Press, pp. 51-60.

Bødker, S. (1991). Through the Interface. A Human Activity Approach to User Interface Design. Mahwah, NJ, USA: Lawrence Erlbaum Associates, Inc.

Bødker, S. \& Petersen, M. G. (2001). Design for learning in use. Scand. J. Inf. Syst. 12, 1-2, pp. 61-80.

Engeström, Y. (1987). Learning by expansion. Helsinki: Orienta Konsultit.

Gal'perin, P. Y. (1969). Stages in the development of mental acts. In Cole, M. \& Maltzman, I. (Eds.). A handbook of contemporary soviet psychology, New York: Basic Books, pp. 249-273.

Kaptelinin, V. (1995). Computer-Mediated Activity: Functional Organs in Social and Developmental Contexts. In Nardi, B. (Ed.). Context and consciousness: activity theory and human-computer interaction, chapter 3, Cambridge, MA: MIT Press, pp. 45-68.

Nielsen, C. \& Søndergaard, A. (2000). Designing for mobility: an integration approach to support mobile technologies. In Proceedings of NordiCHI 2000, CDROM.

Shneiderman, B. \& Plaisant, C. (2005). Designing the User Interface. $4^{\text {th }}$ edition, Addison Wesley 